$>8$ hours. Taste disturbance was the most common adverse event, reported in up to $30 \%$ of patients. (Winner P, Rothner AD, Saper J et al. A randomized, double-blind, placebo-controlled study of sumatriptan nasal spray in the treatment of acute migraine in adolescents. Pediatrics November 2000;106:989-997). (Respond: Paul Winner DO, 5205 Greenwood Avenue, Suite 200, West Palm Beach, FL 33407).

COMMENT. Sumatriptan nasal spray may be an effective and well-tolerated treatment of acute migraine in adolescents. In this large multicenter study, the 20 $\mathrm{mg}$ dose was most effective, especially in older patients.

Cognitive functioning during a migraine attack. A significant loss of cognitive efficiency was demonstrated in 10 adult migraineurs during a migraine attack, in a study at the Headache Care Center, Springfield, MO. (Farmer $\mathrm{K}$ et al. A pilot study to measure cognitive efficiency during migraine. Headache Sept 2000;40:657-661). Recovery of cognitive function followed within 15 minutes of sumatriptan injection $(6 \mathrm{mg})$ and continued to improve at 45 minutes. The adverse impact of cognitive effects of migraine on work and study efficiency is an important consideration in a decision to treat aggressively and prophylactically.

\title{
DIVALPROEX SODIUM FOR PREVENTION OF MIGRAINE
}

The use of divalproex sodium (DVS) as a prophylactic treatment for migraine was studied in 42 patients, 7 to 16 years of age (mean age, 11.3 years) seen between July 1996 and December 1998 at St Jude Children's Research Hospital, Memphis, TN; and Hasbro Children's Hospital/Rhode Island Hospital,Divalproex Brown University, Providence, RI. After 4 months treatment with DVS in doses ranging from 15 to $45 \mathrm{mg} / \mathrm{kg}$.day, $10 \%$ became headache-free, $14 \%$ had a $75 \%$ reduction. $78 \%$ a $50 \%$ reduction in headaches, and $80 \%$ were able to discontinue medicines used to abort attacks. Adverse effects included gastrointestinal upset, weight gain, somnolence, dizzinesss, and tremor. None had hepatotoxicity. (Caruso JM, Brown WD, Exil G, Gascon GG. The efficacy of divalproex sodium in the prophylactic treatment of children with migraine. Headache Sept 2000;40:672676). (Respond: Dr Generoso G Gascon, Pediatric Neurology, Hasbro Children's Hospital/Rhode Island Hospital, 110 Lockwood Street, Suite 342, Providence, RI).

COMMENT. In this uncontrolled trial, divalproex sodium was effective as a prophylactic therapy for childhood migraine.

\section{NEUROIMAGING IN EVALUATION OF MIGRAINE HEADACHE}

The utility of neuroimaging in the evaluation of children with migraine and chronic daily headache, with normal neurologic examination, was determined by a retrospective review of 302 patients, aged between 6 and 18 years, seen in the Pediatric Neurology Clinic, Children's Hospital, Eastern Virginia Medical School, Norfolk, 1997-1999. Other types of headache among the patients reviewed included: migrainelike symptoms (10\%), chronic daily headache and abnormal neurologic examination (2\%), secondary headache $(17 \%)$, complicated migraine $(7 \%)$, posttraumatic headache $(7 \%)$, seizure-related headache $(4 \%)$, brain tumors $(3.6 \%)$, tension-type headache $(3 \%)$, and pseudotumor cerebri $(1.3 \%)$.

Of 107 (35\% of total) with uncomplicated migraine, 42 (39\%) received CT scans, and $2(5 \%)$ were abnormal (arachnoid cyst in 1 and dilated Virschow-Robin space in 1). Of 12 (11\%) who received an MRI, 2 (17\%) were abnormal, both having a Chiari type 1 malformation. Of 30 patients with chronic daily headache, 